\title{
Front Matter: Volume 7953
}

, "Front Matter: Volume 7953," Proc. SPIE 7953, Novel In-Plane

Semiconductor Lasers X, 795301 (10 March 2011); doi: 10.1117/12.890416

SPIE. Event: SPIE OPTO, 2011, San Francisco, California, United States 


\title{
PROCEEDINGS OF SPIE
}

\section{Novel In-Plane Semiconductor Lasers $X$}

\author{
Alexey A. Belyanin \\ Peter M. Smowton \\ Editors
}

25-28 January 2011

San Francisco, California, United States

Sponsored and Published by

SPIE 
The papers included in this volume were part of the technical conference cited on the cover and title page. Papers were selected and subject to review by the editors and conference program committee. Some conference presentations may not be available for publication. The papers published in these proceedings reflect the work and thoughts of the authors and are published herein as submitted. The publisher is not responsible for the validity of the information or for any outcomes resulting from reliance thereon.

Please use the following format to cite material from this book:

Author(s), "Title of Paper," in Novel In-Plane Semiconductor Lasers X, edited by Alexey A. Belyanin, Peter M. Smowton, Proceedings of SPIE Vol. 7953 (SPIE, Bellingham, WA, 2011) Article CID Number.

ISSN 0277-786X

ISBN 9780819484901

Published by

SPIE

P.O. Box 10, Bellingham, Washington 98227-0010 USA

Telephone +1 3606763290 (Pacific Time) · Fax +1 3606471445

SPIE.org

Copyright () 2011, Society of Photo-Optical Instrumentation Engineers

Copying of material in this book for internal or personal use, or for the internal or personal use of specific clients, beyond the fair use provisions granted by the U.S. Copyright Law is authorized by SPIE subject to payment of copying fees. The Transactional Reporting Service base fee for this volume is $\$ 18.00$ per article (or portion thereof), which should be paid directly to the Copyright Clearance Center (CCC), 222 Rosewood Drive, Danvers, MA 01923. Payment may also be made electronically through CCC Online at copyright.com. Other copying for republication, resale, advertising or promotion, or any form of systematic or multiple reproduction of any material in this book is prohibited except with permission in writing from the publisher. The CCC fee code is 0277-786X/11/ \$18.00.

Printed in the United States of America.

Publication of record for individual papers is online in the SPIE Digital Library.

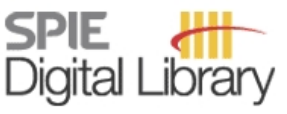

SPIEDigitalLibrary.org

Paper Numbering: Proceedings of SPIE follow an e-First publication model, with papers published first online and then in print and on CD-ROM. Papers are published as they are submitted and meet publication criteria. A unique, consistent, permanent citation identifier (CID) number is assigned to each article at the time of the first publication. Utilization of CIDs allows articles to be fully citable as soon they are published online, and connects the same identifier to all online, print, and electronic versions of the publication. SPIE uses a six-digit CID article numbering system in which:

- The first four digits correspond to the SPIE volume number.

- The last two digits indicate publication order within the volume using a Base 36 numbering system employing both numerals and letters. These two-number sets start with 00, 01, 02, 03, 04 , $05,06,07,08,09,0 A, 0 B \ldots$. OZ, followed by 10-1Z, 20-2Z, etc.

The CID number appears on each page of the manuscript. The complete citation is used on the first page, and an abbreviated version on subsequent pages. Numbers in the index correspond to the last two digits of the six-digit CID number. 


\section{Contents}

LOW DIMENSIONAL MATERIALS

795302 Quantum dot composite light sources [7953-01]

D. W. Grund, Jr., B. C. Olbricht, D. W. Prather, Univ. of Delaware (United States)

795303 Gain and absorption characteristics of bilayer quantum dot lasers beyond $1.3 \mu \mathrm{m}$ [7953-02] M. A. Majid, S. C. Chen, D. T. D. Childs, H. Shahid, R. J. Airey, K. Kennedy, R. A. Hogg, The Univ. of Sheffield (United Kingdom); E. Clarke, P. Spencer, R. Murray, Imperial College London (United Kingdom)

795304 Nanopatterned quantum dot active region lasers on InP substrates (Invited Paper) [7953-03] L. J. Mawst, J. H. Park, Y. Huang, J. Kirch, Univ. of Wisconsin-Madison (United States); Y. Sin, B. Foran, The Aerospace Corp. (United States); C.-C. Liu, P. F. Nealey, T. F. Kuech, Univ. of Wisconsin-Madison (United States)

795306 The lateral ambipolar diffusion length in quantum dot lasers [7953-05]

A. Sobiesierski, D. Naidu, P. M. Smowton, Cardiff Univ. (United Kingdom)

\section{GRATING COUPLED}

795308 Deep etched distributed Bragg reflector (DBR) InP/AIGalnP quantum dot lasers [7953-07] S. Shutts, G. Edwards, S. N. Elliott, P. M. Smowton, Cardiff Univ. (United Kingdom); A. B. Krysa, The Univ. of Sheffield (United Kingdom)

795309 Design and device characteristics of index-coupled wafer-level holographically patterned

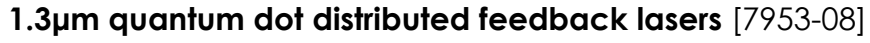

J. Hu, D. Klotzkin, Binghamton Univ. (United States); J. S. Huang, Emcore Broadband (United States); X. Sun, N. Li, Emcore (United States)

7953 OA High power distributed feedback and Fabry-Perot Al-free laser diodes at 780nm for rubidium pumping [7953-09]

C. Cayron, M. Tran, Y. Robert, M. Lecomte, M. Calligaro, O. Parillaud, M. Garcia, M. Krakowski, Alcatel-Thales III-V Lab. (France)

7953 OB Narrow-linewidth distributed feedback lasers with laterally coupled ridge-waveguide surface gratings fabricated using nanoimprint lithography [7953-10]

M. Dumitrescu, J. Telkkälä, J. Karinen, J. Viheriälä, A. Laakso, K. Haring, M.-R. Viljanen, J. Paajaste, R. Koskinen, S. Suomalainen, J. Lyytikäinen, T. Leinonen, M. Pessa, Tampere Univ. of Technology (Finland) 
7953 OD Development of high-speed directly modulated DFB and DBR lasers with surface gratings [7953-12]

M. Dumitrescu, J. Telkkälä, J. Karinen, J. Viheriälä, A. Laakso, Tampere Univ. of Technology (Finland); S. Afzal, J.-P. Reithmaier, Univ. of Kassel (Germany); M. Kamp, Julius-Maximilians-Univ. Würzburg (Germany); P. Melanen, P. Uusimaa, Modulight, Inc. (Finland); P. Bardella, M. Vallone, I. Montrosset, Politecnico di Torino (Italy); O. Parillaud, M. Krakowski, Alcatel Thales III-V Lab. (France); D. Gready, G. Eisenstein, Technion (Israel); G. Sek, Wroclaw Univ. of Technology (Poland)

$7953 \mathrm{OE} \quad 1.55 \mu \mathrm{m}$ directly modulated CCIG lasers fabricated by surface-defined lateral feedback gratings [7953-13]

S. Afzal, F. Schnabel, W. Scholz, J.-P. Reithmaier, Univ. of Kassel (Germany); G. Eisenstein,

D. Gready, Technion (Israel); O. Parillaud, M. Krakowski, Alcatel-Thales III-V Lab. (France);

I. Montrosset, M. Vallone, Politecnico di Torino (Italy)

7953 OF $\quad 40$ Gbit/s directly modulated lasers: physics and application (Invited Paper) [7953-14] U. Troppenz, J. Kreissl, M. Möhrle, C. Bornholdt, W. Rehbein, B. Sartorius, Fraunhofer Institute for Telecommunications (Germany); I. Woods, Cogo Optronics Inc. (United States); M. Schell, Fraunhofer Institute for Telecommunications (Germany)

\section{NITRIDES}

7953 OG Recent results of blue and green InGaN laser diodes for laser projection (Invited Paper) [7953-15]

S. Lutgen, D. Dini, I. Pietzonka, S. Tautz, A. Breidenassel, A. Lell, A. Avramescu, C. Eichler, T. Lermer, J. Müller, G. Bruederl, A. Gomez-Iglesias, U. Strauss, OSRAM Opto Semiconductors GmbH (Germany); W. G. Scheibenzuber, U. T. Schwarz, Fraunhofer IAF (Germany); B. Pasenow, S. Koch, Univ. Marburg (Germany)

$7953 \mathrm{OH} \quad$ Gain characteristics of deep UV AIGaN quantum wells lasers [7953-16]

J. Zhang, H. Zhao, N. Tansu, Lehigh Univ. (United States)

7953 Ol Plasmonic cladding InGaN MQW laser diodes (Invited Paper) [7953-17] P. Perlin, K. Holc, M. Sarzyński, M. Leszczyński, R. Czernecki, Institute of High Pressure Physics (Poland) and TopGaN Ltd. (Poland); Ł. Marona, Institute of High Pressure Physics (Poland); P. Wisniewski, Institute of High Pressure Physics (Poland) and TopGaN Ltd. (Poland); G. Cywinski, C. Skierbiszewski, M. Bockowski, I. Grzegory, T. Suski, Institute of High Pressure Physics (Poland)

7953 0J High peak power picoseconds optical pulse generation from GalnN semiconductor diode lasers (Invited Paper) [7953-18]

R. Koda, Tohoku Univ. (Japan) and Sony Corp. (Japan); T. Oki, Sony Corp. (Japan); T. Miyajima, Tohoku. Univ. (Japan) and Sony Corp. (Japan); H. Watanabe, Sony Corp. (Japan); S. Kono, Tohoku Univ. (Japan); M. Kuramoto, M. Ikeda, Tohoku Univ. (Japan) and Sony Corp. (Japan); H. Yokoyama, Tohoku Univ. (Japan)

7953 OK Dynamics of GaN-based laser diodes from violet to green [7953-19] W. G. Scheibenzuber, C. Hornuss, Fraunhofer IAF (Germany); U. T. Schwarz, Fraunhofer IAF (Germany) and Freiburg Univ. (Germany) 
7953 ON The temperature dependence of key electro-optical characteristics for mid-infrared emitting quantum cascade lasers (Invited Paper) [7953-22]

D. Botez, J. C. Shin, Univ. of Wisconsin-Madison (United States); S. Kumar, Lehigh Univ. (United States); J. Kirch, C.-C. Chang, L. J. Mawst, Univ. of Wisconsin-Madison (United States);

I. Vurgaftman, J. R. Meyer, U.S. Naval Research Lab. (United States); A. Bismuto, B. Hinkov,

J. Faist, ETH Zurich (Switzerland)

\section{THz QUANTUM CASCADE LASERS I}

795300 High-temperature performance and broad continuous tunability of terahertz quantum-cascade lasers (Invited Paper) [7953-23]

S. Kumar, Lehigh Univ. (United States); Q. Qin, C. W. I. Chan, Q. Hu, Massachusetts Institute of Technology (United States); J. L. Reno, Sandia National Labs. (United States)

7953 OP Broadband semiconductor terahertz laser based on heterogeneous cascades [7953-24] D. Turčinková, G. Scalari, M. I. Amanti, F. Castellano, M. Beck, J. Lloyd-Hughes, J. Faist, ETH Zurich (Switzerland)

\section{THz LASERS II}

7953 OS Monolithically integrated THz transceivers (Invited Paper) [7953-27]

M. C. Wanke, M. Lee, C. D. Nordquist, M. J. Cich, Sandia National Labs. (United States); A. D. Grine, LMATA Government Services (United States); C. T. Fuller, J. L. Reno, Sandia National Labs. (United States)

7953 OT Stimulated Smith-Purcell semiconductor THz sources [7953-28]

D. D. Smith, A. Belyanin, Texas A\&M Univ. (United States)

7953 OU Upper limits on terahertz difference frequency generation power in quantum well heterostructures [7953-29]

Y.-H. Cho, Texas A\&M Univ. (United States); M. A. Belkin, The Univ. of Texas at Austin (United States); A. Belyanin, Texas A\&M Univ. (United States)

\section{HIGH POWER I}

$79530 Z \quad 670 \mathrm{~nm}$ nearly diffraction limited tapered lasers with more than $30 \%$ conversion efficiency and $1 \mathrm{~W}$ cW and $3 \mathrm{~W}$ pulsed output power [7953-34]

B. Sumpf, P. Adamiec, M. Zorn, H. Wenzel, G. Erbert, G. Tränkle, Ferdinand-Braun-Institut für Höchstfrequenztechnik (Germany)

795310 Versatile $1 \mathrm{~W}$ narrow band $976 \mathrm{~nm}$ and $1064 \mathrm{~nm}$ light sources [7953-35]

S. Mohrdiek, H.-U. Pfeiffer, E. A. Zibik, B. Sverdlov, T. Pliska, N. Lichtenstein, Oclaro Switzerland AG (Switzerland) 
$795311 \quad 1 \mathrm{~W}$ semiconductor based laser module with a narrow linewidth emitting near $1064 \mathbf{n m}$ [7953-36]

S. Spießberger, Ferdinand-Braun-Institut, Leibniz-Institut für Höchstfrequenztechnik (Germany); M. Schiemangk, Humboldt-Univ. zu Berlin (Germany); A. Sahm, A. Wicht, H. Wenzel, J. Fricke, G. Erbert, Ferdinand-Braun-Institut, Leibniz-Institut für Höchstfrequenztechnik (Germany)

\section{MID-INFRARED LASERS}

795314 Room-temperature 4.0- $\mu \mathrm{m}$ broadened optical pumping injection cavity lasers [7953-39] L. J. Olafsen, Baylor Univ. (United States); L. E. Bain, Baylor Univ. (United States) and Univ. of North Carolina, Chapel Hill (United States); W. W. Bewley, I. Vurgaftman, J. R. Meyer, U.S. Naval Research Lab. (United States); H. Lee, Sarnoff Corp. (United States) and Applied Optoelectronics, Inc. (United States); R. U. Martinelli, Sarnoff Corp. (United States)

795315 InGaAs/AllnAs quantum cascade laser sources based on intra-cavity second harmonic generation emitting in 2.6-3.6 micron range (Invited Paper) [7953-40]

M. A. Belkin, M. Jang, R. W. Adams, The Univ. of Texas at Austin (United States); J. X. Chen, W. O. Charles, C. Gmachl, Princeton Univ. (United States); L. W. Cheng, F.-S. Choa, Univ. of Maryland, Baltimore County (United States); X. Wang, M. Troccoli, AdTech Optics, Inc. (United States); A. Vizbaras, M. Anders, C. Grasse, M.-C. Amann, Walter Schottky Institute (Germany)

MID-INFRARED QCLS

795317 High performance quantum cascade lasers with broadband gain spectra (Invited Paper) [7953-42]

Y. Yao, Princeton Univ. (United States); X. Wang, J.-Y. Fan, AdTech Optics, Inc. (United States); W. O. Charles, T. Tsai, J. Chen, G. Wysocki, C. F. Gmachl, Princeton Univ. (United States)

$79531 \mathrm{~A} \quad 3.5 \mu \mathrm{m}$ strain balanced GalnAs/AllnAs quantum cascade lasers operating at room temperature [7953-71]

F. Xie, C. Caneau, H. P. LeBlanc, N. J. Visovsky, Corning Inc.(United States);

Y. Wang, G. Wysocki, Princeton Univ. (United States); L. C. Hughes, C. Zah, Corning Inc. (United States)

\section{HIGH POWER II}

7953 1B Performance and reliability of high power 7xx $\mathbf{n m}$ laser diodes (Invited Paper) [7953-45] L. Bao, J. Wang, M. Devito, D. Xu, M. Grimshaw, W. Dong, X. Guan, H. Huang, P. Leisher, S. Zhang, D. Wise, R. Martinsen, J. Haden, nLIGHT Corp. (United States)

7953 1C Very high modulation efficiency two-sections tapered laser diode at $1060 \mathrm{~nm}$ for free space optical communications [7953-46]

M. Ruiz, N. Michel, M. Calligaro, Y. Robert, M. Lecomte, O. Parillaud, M. Krakowski, Alcatel-Thales III-V Lab. (France); I. Esquivias, H. Odriozola, J. M. G. Tijero, Univ. Politécnica de Madrid (Spain); C. H. Kwok, R. V. Penty, I. H. White, Univ. of Cambridge (United Kingdom) 
7953 ID Compact ps-pulse laser source with free adjustable repetition rate and $\mathrm{nJ}$ pulse energy on microbench [7953-47]

A. Klehr, A. Liero, T. Hoffmann, S. Schwertfeger, H. Wenzel, G. Erbert, W. Heinrich, G. Tränkle, Ferdinand-Braun-Institut, Leibniz-Institut für Höchstfrequenztechnik (Germany)

7953 IE Low-cost high-reliability 830nm single mode lasers for consumer electronics and CtP applications [7953-48]

J. Boucart, E. Zibik, S. Renz, B. Sverdlov, Oclaro Switzerland AG (Switzerland); M. Kearley,

D. Inder, C. Button, Oclaro PLC (United Kingdom); N. Lichtenstein, Oclaro Switzerland AG

(Switzerland)

HIGH POWER III

7953 IF Mitigation of thermal lensing effect as a brightness limitation of high-power broad area diode lasers [7953-49]

J. G. Bai, P. Leisher, S. Zhang, S. Elim, M. Grimshaw, C. Bai, L. Bintz, D. Dawson, L. Bao,

J. Wang, M. DeVito, R. Martinsen, J. Haden, nLIGHT Corp. (United States)

7953 1G Reliable operation of $976 \mathrm{~nm}$ high power DFB broad area diode lasers with over $60 \%$ power conversion efficiency (Invited Paper) [7953-50]

P. Crump, C. M. Schultz, H. Wenzel, S. Knigge, O. Brox, A. Maaßdorf, F. Bugge, G. Erbert,

Ferdinand-Braun-Institut, Leibniz-Institut für Höchstfrequenztechnik (Germany)

$795311 \quad$ A novel approach to finite-aperture tapered unstable resonator lasers [7953-52]

M. Spreemann, H. Wenzel, B. Eppich, Ferdinand-Braun-Institut, Leibniz-Institut für

Höchstfrequenztechnik (Germany); M. Lichtner, Weierstrass Institute for Applied Analysis and Stochastics (Germany); G. Erbert, Ferdinand-Braun-Institut, Leibniz-Institut für

Höchstfrequenztechnik (Germany)

7953 i Control of slow axis mode behaviour with waveguide phase structures in semiconductor broad-area lasers [7953-53]

H.-C. Eckstein, U. D. Zeitner, K. Ahmed, Fraunhofer Institute for Applied Optics and Precision

Engineering (Germany); W. Schmid, U. Strauss, OSRAM Opto Semiconductors (Germany)

MID-IR LASERS AND APPLICATIONS I

7953 IK Recent results from broadly tunable external cavity quantum cascade lasers (Invited Paper) [7953-54]

D. Caffey, M. B. Radunsky, V. Cook, M. Weida, P. R. Buerki, S. Crivello, T. Day, Daylight

Solutions (United States)

7953 IL Multi-watt level short wavelength quantum cascade lasers [7953-55]

A. Lyakh, R. Maulini, A. G. Tsekoun, R. Go, C. K. N. Patel, Pranalytica, Inc. (United States)

MID-IR LASERS AND APPLICATIONS II

7953 IP Design and operation of mid-IR integrated DBR tunable lasers [7953-59]

L. Cheng, F.-S. Choa, Univ. of Maryland, Baltimore County (United States) 
$79531 Q \quad$ Type-I GaSb based single lateral mode diode ridge lasers operating at room temperature in 3.1-3.2 $\mu \mathrm{m}$ spectral region [7953-60]

G. Tsvid, T. Hosoda, J. Chen, G. Kipshidze, L. Shterengas, State Univ. of New York at Stony Brook (United States); C. Frez, A. Soibel, S. Forouhar, Jet Propulsion Lab. (United States);

G. Belenky, State Univ. of New York at Stony Brook (United States)

7953 IR Broad area lasers with folded-resonator geometry for integrated transverse mode selection [7953-61]

D. Hoffmann, K. Huthmacher, C. Doering, H. Fouckhardt, Kaiserslautern Univ. of Technology (Germany)

7953 is Near-infrared induced negative photoconductance and its relationship with optical quenching of mid-infrared quantum cascade lasers [7953-62]

D. Guo, F.-S. Choa, L. Cheng, X. Chen, Univ. of Maryland, Baltimore County (United States)

7953 IT Novel mid-IR quantum cascade laser waveguide coupling techniques [7953-63]

D. Shyu, F.-S. Choa, X. Chen, Univ. of Maryland, Baltimore County (United States); S. Trivedi, Brimrose Corp. (United States)

\section{POSTER SESSION}

7953 IW Comparison of gain measurement techniques for $1.3 \mu \mathrm{m}$ quantum dot lasers [7953-65] H. Shahid, D. T. D. Childs, B. J. Stevens, R. A. Hogg, The Univ. of Sheffield (United Kingdom)

$79531 \mathrm{X} \quad$ Optical gain in erbium lithium niobate titanium diffused waveguides [7953-66]

G. A. Ejzak, D. W. Prather, Univ. of Delaware (United States)

$79531 \mathrm{Y} \quad$ Controlled intermixing of multiple quantum wells for broadly tunable integrated lasers [7953-67]

A. J. Zakariya, CREOL, The College of Optics and Photonics, Univ. of Central Florida (United States), Univ. of Central Florida (United States), and Information Technology \& Communication Sector, MOl-Kuwait (Kuwait); N. Bickel, Univ. of Central Florida (United States); P. LiKamWa, CREOL, The College of Optics and Photonics, Univ. of Central Florida (United States) and Univ. of Central Florida (United States)

795312 Low threshold short cavity quantum cascade lasers [7953-69]

X. Chen, L. Cheng, D. Guo, F.-S. Choa, T. Worchesky, Univ. of Maryland, Baltimore County (United States)

795320 Thermal investigation of mid-infrared quantum cascade lasers under quasi-continuous-wave operations [7953-70]

X. Chen, L. Cheng, D. Guo, F.-S. Choa, T. Worchesky, Univ. of Maryland, Baltimore County (United States)

Author Index 


\title{
Conference Committee
}

\author{
Symposium Chair \\ Liang-Chy Chien, Kent State University (United States) \\ Symposium Cochairs
}

Klaus P. Streubel, OSRAM GmbH (Germany)

E. Fred Schubert, Rensselaer Polytechnic Institute (United States)

Program Track Chair

Klaus P. Streubel, OSRAM GmbH (Germany)

Conference Chairs

Alexey A. Belyanin, Texas A\&M University (United States)

Peter M. Smowton, Cardiff University (United Kingdom)

Program Committee

Martin Achtenhagen, Photodigm, Inc. (United States)

Yasuhiko Arakawa, The University of Tokyo (Japan)

Dan Botez, University of Wisconsin-Madison (United States)

David Bour, Palo Alto Research Center, Inc. (United States)

Federico Capasso, Harvard University (United States)

Claire F. Gmachl, Princeton University (United States)

Michael Kneissl, Technische Universität Berlin (Germany)

Hui Chun Liu, National Research Council Canada (Canada)

Luke J. Mawst, University of Wisconsin-Madison (United States)

Jerry R. Meyer, U.S. Naval Research Laboratory (United States)

Jesper Moerk, Technical University of Denmark (Denmark)

Mario J. Paniccia, Intel Corporation (United States)

Richard V. Penty, University of Cambridge (United Kingdom)

Johann-Peter Reithmaier, Universität Kassel (Germany)

Nelson Tansu, Lehigh University (United States)

Session Chairs

1 Low Dimensional Materials

Johann-Peter Reithmaier, Universität Kassel (Germany) 
2 Grating Coupled

Luke J. Mawst, University of Wisconsin-Madison (United States)

3 Telecom/Datacom

Richard V. Penty, University of Cambridge (United Kingdom)

$4 \quad$ Nitrides

Michael Kneissl, Technische Universität Berlin (Germany)

5 Quantum Cascade Lasers I

Yu Yao, Princeton University (United States)

6 THz Quantum Cascade Lasers I

Mikhail A. Belkin, The University of Texas at Austin (United States)

$7 \quad$ THz Lasers II

Nanfang Yu, Harvard University (United States)

8 Silicon Photonics: Joint Session with Conference 7943

Haisheng Rong, Intel Corporation (United States)

9 High Power I

Paul O. Leisher, nLIGHT Corporation (United States)

10 Mid-infrared Lasers

Dan Botez, University of Wisconsin-Madison (United States)

11 Mid-infrared QCLS

Jerry R. Meyer, U.S. Naval Research Laboratory (United States)

12 High Power II

Gary A. Evans, Southern Methodist University (United States)

13 High Power III

Gary A. Evans, Southern Methodist University (United States)

14 Mid-IR Lasers and Applications I

Mariano Troccoli, AdTech Optics, Inc. (United States)

15 Mid-IR Lasers and Applications II

Stefan Menzel, Harvard University (United States) 